\title{
Persistent transitional circulation
}

\author{
RAYMOND BROWN and DOUGLAS PICKERING \\ From Radcliffe Infirmary, Oxford
}

\begin{abstract}
Brown, R., and Pickering, D. (1974). Archives of Disease in Childhood, 49, 883. Persistent transitional circulation. Six infants presented within 24 hours of birth with cyanosis. The clinical findings, combined with catheter studies in 4 cases, led to the conclusion that there was a persistent fetal direction of ductus arteriosus flow with pulmonary hypertension, i.e. a 'persistent transitional circulation'. The occurrence was $1 / 1454$ live births, and it is thus an important condition to recognize and differentiate from cyanotic congenital heart disease. 4 of the children went through the transition to a normal infant circulation with medical treatment alone, one died before this occurred, and one recovered after an exchange transfusion. The cause of the persistent transitional circulation is not clear, but it is probably the end result of several different pathological processes.
\end{abstract}

Persistent fetal direction of ductus arteriosus flow with pulmonary hypertension is important in the differential diagnosis of neonatal cyanotic congenital heart disease (Rowe, 1970). 6 cases of this syndrome are described below and the typical picture, causes, and treatment are discussed.

\section{Case reports}

Case 1. This boy was born to an unmarried 20-yearold primipara who had taken cannabis and LSD during early pregnancy, but whose pregnancy was otherwise uneventful. Labour began at term, was normal, and proceeded to normal delivery in a General Practitioner Unit. The baby weighed $3880 \mathrm{~g}$, cried immediately, but remained hypotonic and deeply cyanosed. He failed to improve with facial mask oxygen and at $\mathbf{1 5}$ minutes was still cyanosed and had developed grunting respirations, the heart rate falling to $100 / \mathrm{min}$. A paediatrician was called; the infant was intubated and ventilated with $100 \%$ oxygen, without lessening of the cyanosis. There were signs of cardiac failure, with peripheral oedema and bilateral basal crepitations. Blood-stained frothy mucus was aspirated from the trachea. No cardiac murmur was heard and the peripheral pulses were of 'full' volume. Chest $x$-ray showed diffuse reticular shadowing throughout both lung fields and a small left apical pneumothorax, but the cardiothoracic ratio was normal. ECG showed an axis of $160^{\circ}$ with some clock-wise rotation only.

At $1 \frac{1}{2}$ hours umbilical arterial and venous catheters were inserted and arterial blood gases showed $p \mathrm{H} 7 \cdot 22$, $\mathrm{PaO}_{2} 29 \mathrm{mmHg}, \mathrm{PaCO}_{2} 27 \mathrm{mmHg}$, and base deficit of 15 $\mathrm{mEq} / \mathrm{l}$. when ventilated with $100 \%$ oxygen at a pressure

Received 10 April 1974. of $4 \mathrm{cmH}_{2} \mathrm{O}$. The heart failure responded to treatment with digoxin and frusemide. However, the arterial $\mathrm{PaO}_{2}$ never rose above $30 \mathrm{mmHg}$ and progressive acidosis developed which initially responded to sodium bicarbonate infusion but which eventually became intractible; the baby died 16 hours after birth. At necropsy there was pulmonary congestion and a large persistent ductus arteriosus, but brain, heart, and great vessels were normal. Lung histology showed no evidence of infection, hyaline membranes, or focal alveolar atelectasis, but there was striking dilatation of the subpleural and perivascular lymphatics.

Case 2. This boy was the third baby born to a mother who had had 2 previous children, one of whom had died of congenital heart disease. The pregnancy was normal, ending at 37 weeks with a lower segment caesarean section because of a previous section. Birthweight was $3060 \mathrm{~g}$. Facial oxygen and mucus extraction caused him to gasp at 30 seconds and Apgar score at one minute was 5. A bradycardia of $40 / \mathrm{min}$ developed and the child was transferred to the Intensive Care Unit. Physical examination showed a centrally cyanosed child who responded to the raised inspired oxygen concentration by becoming pink. The pulse rate was $110 / \mathrm{min}$, respiratory rate $90 / \mathrm{min}$. There was marked indrawing of the chest wall with inspiration but no evidence of cardiac failure. A soft systolic murmur was noted at the lower left sternal border on one occasion only. Examination of peripheral pulses, chest $x$-ray, and ECG was normal. At 1 hour blood gases with the child in $42 \%$ oxygen showed $p \mathrm{H} \mathrm{7} \cdot 36, \mathrm{PaO}_{2} 54 \mathrm{mmHg}$, and $\mathrm{PaCO}_{2}$ $28 \mathrm{mmHg}$. Cardiac catheterization at 36 hours of age showed a large persistent ductus arteriosus with bidirectional shunting and a bidirectional shunt through 
the patent foramen ovale. The pulmonary arterial pressure was $60 / 30 \mathrm{mmHg}$ (mean 40 ), pulmonary arterial oxygen saturation $27 \%$, aortic pressure $52 / 30 \mathrm{mmHg}$ (mean 38), oxygen saturation $38 \%$. There was no abnormality of the left heart. The child was ventilated using an Engstrom respirator with $100 \%$ oxygen and positive expiratory airways pressure. His condition steadily improved and he was transferred back to the maternity department, aged 48 hours, with no evidence of lung or cardiac abrormality.

Case 3. This girl was born at term, forceps delivery. She breathed at once, but remained persistently cyanosed. Physical examination showed a sick, centrally cyanosed infant with marked tachypnoea and moderate tachycardia. The femoral pulses were normal and the liver enlarged $2 \mathrm{~cm}$ below the right costal margin. $\mathrm{Hb}$ was $18.9 \mathrm{~g} / 100 \mathrm{ml}$, chest $x$-ray and ECG were normal. Cardiac catheterization was carried out on the second day of life and showed a right-to-left shunt at atrial level and also at great artery level via a large persistent ductus arteriosus. Pulmonary and systemic artery pressures were similar, the pulmonary and cardiac outputs were equal, the main pulmonary arterial pressure was $45 / 20$ $\mathrm{mmHg}$, abdominal aortic pressure $43 / 35 \mathrm{mmHg}$, right atrial mean pressure $2 \mathrm{mmHg}$, and left atrial mean pressure $8 \mathrm{mmHg}$. Angiocardiography showed a large persistent ductus arteriosus and no other significant cardiac abnormality. The child was nursed in an incubator containing $40 \%$ oxygen and over the next 3 days her cyanosis, tachypnoea, and tachycardia subsided. When seen at follow-up at the age of 1 year she was growing and developing normally.

Case 4. This boy was a second child born at 42 weeks' gestation; pregnancy was normal but the second stage was prolonged with fetal distress, the child passing meconium and the fetal heart rate falling to $80 / \mathrm{min}$. He was found to be in a persistent occipitoposterior position, but was delivered spontaneously. He weighed $3620 \mathrm{~g}$ and suffered from primary asphyxia initially. Intubation was performed; he was ventilated for 4 minutes after birth and was subsequently given oxygen by a face mask. The child remained persistently and markedly cyanosed but there was no respiratory distress and no evidence of cardiac failure. There were slightly weak peripheral pulses in all areas and a grade $1 / 4$ systolic murmur was heard at the lower left sternal border. The chest was clinically clear. Chest $x$-ray showed a slight increase in vascularity only. ECG in the first 24 hours was normal. $\mathrm{He}$ was nursed in an incubator containing $35 \%$ oxygen and the central cyanosis persisted until, at the end of 72 hours, he suddenly became pink and the cardiac murmur disappeared. The child has remained normal on subsequent follow-up at 5 months.

Case 5. This boy, the first child of a healthy mother, was born at 42 weeks' gestation after a normal pregnancy, but forceps were applied for fetal distress. He weighed $3370 \mathrm{~g}$ and his condition at birth was recorded as 'poor'.
Physical examination showed a centrally cyanosed child with respiratory distress, but with no heart murmurs nor cardiac failure. The peripheral pulses were normal. Chest $x$-ray showed a slight increase in cardiothoracic ratio and some possible right upper lobe collapse. ECG was normal apart from an axis of $190^{\circ}$. Cardiac catheterization was carried out on the third day and showed a pulmonary arterial pressure of $48 / 12 \mathrm{mmHg}$ (mean 34); pulmonary arterial oxygen saturation was $10 \%$, aortic pressure $40 / 25 \mathrm{mmHg}$ (mean 30 ), and arterial oxygen saturation $22 \%$. Angiocardiography showed a large persistent ductus arteriosus and a normal left heart. The child was ventilated with $100 \%$ oxygen using an Engstrom ventilator and positive expiratory airways pressure. The clinical condition continued to deteriorate and so an exchange transfusion was performed in an attempt to replace fetal by adult haemoglobin and thus transport more oxygen to the pulmonary arteriolar walls, and also to wash out any humoral factor that may have caused persistent pulmonary arteriolar spasm. At the end of exchange transfusion the child's condition was extremely poor but he made a steady recovery over the next 36 hours. At the age of 4 months the infant was developing normally with no signs of lung or heart disease.

Case 6. This boy was born at 38 weeks' gestation to a 20-year-old mother, her second child, after a normal pregnancy. Labour was uneventful. Birthweight was $3860 \mathrm{~g}$. He was apnoeic and cyanosed at birth and required intubation and intermittent positive pressure ventilation. $\mathrm{He}$ was noted to be cyanosed after extubation and this was only partially relieved by increasing the ambient oxygen concentration. At one hour of age he was noted to be tachypnoeic, with grunting and increased cyanosis. A short ejection systolic murmur was heard. A radial artery sample taken at 20 hours in $100 \%$ oxygen revealed a $p \mathrm{H}$ of $7 \cdot 23$, $\mathrm{PaCO}_{2}$ of 46, and $\mathrm{PaO}_{2}$ of $66 \mathrm{mmHg}$. At 30 hours umbilical arterial catheterization was performed, the $\mathrm{PaO}_{2}$ in $100 \%$ oxygen being $43 \mathrm{mmHg}$. $X$-ray of the chest at 3 hours was normal, while that at 30 hours showed bilateral air bronchograms and a 'ground-glass' appearance of the lung fields. He was digitalized and given antibiotics. ECG showed an axis of $+150^{\circ}$ and was otherwise normal. At 72 hours a cardiac catheterization showed a persistent ductus arteriosus, pulmonary hypertension (pulmonary artery pressure of $50 / 40 \mathrm{mmHg}$ ), and a pulmonary venous oxygen saturation of $66 \%$. Just before the catheterization he had a cardiorespiratory arrest requiring resuscitation.

Because of persistently low oxygen tensions, a further 72 hours of positive pressure ventilation was required, the infant being changed over to continuous positive airway pressure (CPAP) when the $\mathrm{PaO}_{2}$ rose to greater than $100 \mathrm{mmHg}$ in $40 \%$ oxygen.

Attempts were made to wean him off the CPAP but this resulted in a recurrence of the cyanosis with severe subcostal recession. On day 13 he was extubated after a period off CPAP and was discharged on day 24 , by which time his tachypnoea had settled. 


\section{Discussion}

Emmanouilides (1973) found a persistent 'fetal' pattern of circulation at $4 \%$ of neonatal cardiac catheterizations. We found 3 infants with this syndrome among 4363 consecutive deliveries at the John Radcliffe Hospital, Oxford, giving an incidence of $1 / 1454$ live births.

Persistent 'fetal' circulation may occur secondary to placental transfusion, polycythaemia, and hyperviscosity (Dunn, 1973; Fouron and Hebert, 1973; Gross, Hathaway, and McGaughey, 1973), hypoglycaemia (Gersony, 1973), clinically unsuspected lung disease (D. Fyler, personal communication, 1973), and early systemic hypoxaemia (Roberton, Hallidie-Smith, and Davis, 1967) and persistent hypoventilation from cerebral causes (Shinebourne, 1974), but in some cases none of these associated conditions is present. None of our infants was significantly polycythaemic or hypoglycaemic. Their clinical illness resembled atypical respiratory distress syndrome in the term infant as described by Roberton et al. (1967).

These were all term infants who were either cyanosed from birth without (in most cases) being apnoeic or else developed cyanosis shortly after birth. The cyanosis was marked, with absent or minimal signs of respiratory distress within the first 24 hours, and usually without the usual $x$-ray findings of the respiratory distress syndrome. The pulses and heart sounds were variable, while murmurs, cardiac failure, and cardiomegaly were sometimes present and sometimes absent. Chest $x$-ray occasionally showed patchy opacities, possibly secondary to aspiration, or patchy atelectasis, but ECG was usually normal, the axis being to the rightward end of the normal range. Cardiac catheterization showed a pulmonary arterial pressure equal to or greater than the systemic, causing a right-to-left or a bidirectional shunt via a persistent ductus arteriosus.

Both right and left heart studies should be carried out in these children to exclude both clinically unsuspected lung disease, as manifested by a low pulmonary venous oxygen saturation (D. Fyler, personal communication, 1973), and pulmonary hypertension secondary to left heart lesions. All 6 of our infants were treated with digoxin plus diuretics, and acidosis was corrected with intra- venous sodium bicarbonate. They were nursed in raised concentrations of oxygen and ventilated if necessary in an attempt to reverse the presumed pulmonary arteriolar constriction. Positive expiratory airways pressure appeared to benefit Cases 2, 5, and 6.

One child (Case 1) died; Cases 2, 3, 4, and 6 recovered with supportive treatment (including ventilation and CPAP when necessary) and Case 5, who was progressively deteriorating, recovered within 24 hours of an exchange transfusion. This may have been coincidental, but the change from fetal to adult haemoglobin or the elimination of 'humoral' substances acting on both ductus and pulmonary arterioles might have been a significant factor in his recovery.

The term 'persistent fetal circulation' (Gersony, 1973) is perhaps a misnomer as the placenta is obviously no longer part of the circulation in these infants. It is suggested that 'persistent transitional circulation' may be a more appropriate descriptive name for this condition until more is learnt of its mechanism (G. S. Dawes, personal communication, 1974).

We thank Professor J. P. M. Tizard and Dr. G. S. Dawes for advice, and Dr. J. Taylor of The Hospital for Sick Children, Great Ormond Street, who catheterized Case 3.

\section{REFERENCES}

Dunn, P. M. (1973). Neonatal Polycythaemia. Paper presented at Neonatal Symposium, Bristol, October 1973.

Emmanouilides, G. C. (1973). Neonatal Cardiorespiratory Distress without Congenital Heart Disease. Paper presented at 'Festschrift' to J. D. Keith, Toronto, May 1973.

Fouron, J. C., and Hebert, F. (1973). The circulatory effects of hematocrit variations in normovolemic newborn lambs. fournal of Pediatrics, 82, 995.

Gersony, W. M. (1973). Persistence of the fetal circulation. A commentary. Fournal of Pediatrics, 82, 1103.

Gross, G. P., Hathaway, W. E., and McGaughey, H. R. (1973). Hyperviscosity in the neonate. Fournal of Pediatrics, 82, 1004

Roberton, N. R. C., Hallidie-Smith, K. A., and Davis, J. A. (1967) Severe respiratory distress syndrome mimicking cyanotic heartdisease in term babies. Lancet, 2, 1108.

Rowe, R. D. (1970). Serious congenital heart disease in the newborn infant: diagnosis and management. Pediatric Clinics of North America, 17, 967.

Shinebourne, E. A. (1974). Growth and development of the cardio-vascular system-functional development. In Scientific Foundations of Paediatrics, p. 198. Ed. by J. A. Davis and J. Dobbing. Heinemann, London.

Correspondence to Dr. D. Pickering, Department of Paediatrics, The Radcliffe Infirmary, Oxford OX2 6HE. 